\title{
New extensions of associated Laguerre polynomials
}

\author{
Ahmed Ali Al-Gonah \\ Communicated by Alexey Lukashov
}

\begin{abstract}
The main object of this paper is to present new extensions of associated Laguerre polynomials. Some integral representations, recurrence relations, generating functions and summation formulae are obtained for these new extended Laguerre polynomials.
\end{abstract}

Keywords. Extended beta function, associated Laguerre polynomials, generating functions, integral formulae.

2020 Mathematics Subject Classification. 33B15,33B20,33C05,33C20,33C45,33C60.

\section{Introduction}

In many areas of applied mathematics, different types of special functions have became necessary tool for the scientists and engineers. During the recent decades or so, various interesting extensions of different special functions such as gamma and beta functions, the Gauss hypergeometric function, and so on have been introduced by several authors (see [1,4,5,13-15]). In 1997, Chaudhry et al. [4] have introduced the extension of Euler's Beta function as follows:

$$
\begin{gathered}
B(x, y ; p)=\int_{0}^{1} t^{x-1}(1-t)^{y-1} \exp \left[\frac{-p}{t(1-t)}\right] d t \\
(\operatorname{Re}(p) \geq 0, \operatorname{Re}(x)>0, \operatorname{Re}(y)>0) .
\end{gathered}
$$

It is clearly seen that $B(x, y ; 0)=B(x, y)$ where $B(x, y)$ is the classical Beta function defined by [16]:

$$
B(x, y)=\int_{0}^{1} t^{x-1}(1-t)^{y-1} d t=\frac{\Gamma(x) \Gamma(y)}{\Gamma(x+y)}, \quad(\operatorname{Re}(x)>0, \operatorname{Re}(y)>0)
$$

where $\Gamma(x)$ is the classical Gamma function (see [16]).

Further, Chaudhry et al. [5] made use of the extended Beta function $B(x, y ; p)$ to extend the Gauss hypergeometric and confluent hypergeometric functions as follows:

$$
F_{p}(a, b ; c ; z)=\sum_{n=0}^{\infty}(a)_{n} \frac{B_{p}(b+n, c-b)}{B(b, c-b)} \frac{z^{n}}{n !}
$$




$$
\begin{gathered}
(\operatorname{Re}(p) \geq 0 ;|z|<1 ; \operatorname{Re}(c)>\operatorname{Re}(b)>0), \\
\Phi_{p}(b ; c ; z)=\sum_{n=0}^{\infty} \frac{B_{p}(b+n, c-b)}{B(b, c-b)} \frac{z^{n}}{n !}, \\
(\operatorname{Re}(p) \geq 0 ; \operatorname{Re}(c)>\operatorname{Re}(b)>0),
\end{gathered}
$$

respectively, where $(a)_{n}$ denotes the Pochhammer's symbol defined in terms of Gamma function by [16]:

$$
(a)_{n}=\frac{\Gamma(a+n)}{\Gamma(a)},(a)_{0}=1 .
$$

Note that

$$
\begin{gathered}
F_{0}(a, b ; c ; z)={ }_{2} F_{1}(a, b ; c ; z), \\
\Phi_{0}(b ; c ; z)=\Phi(b ; c ; z)={ }_{1} F_{1}(b ; c ; z),
\end{gathered}
$$

where ${ }_{2} F_{1}(a, b ; c ; z)$ and ${ }_{1} F_{1}(b ; c ; z)$ ( or $\left.\Phi(b ; c ; z)\right)$ are the classical Gauss hypergeometric and confluent hypergeometric functions respectively which are special cases of the generalized hypergeometric function defined as [16]:

$$
{ }_{r} F_{s}\left[\begin{array}{c}
\alpha_{1}, \alpha_{2}, \ldots, \alpha_{r} ; \\
\beta_{1}, \beta_{2}, \ldots, \beta_{s} ;
\end{array}\right]=\sum_{n=0}^{\infty} \frac{\left(\alpha_{1}\right)_{n}\left(\alpha_{2}\right)_{n} \ldots\left(\alpha_{r}\right)_{n}}{\left(\beta_{1}\right)_{n}\left(\beta_{2}\right)_{n} \ldots\left(\beta_{s}\right)_{n}} \frac{z^{n}}{n !} .
$$

Recently, the extended Beta function $B(x, y ; p)$ and its systemic generalizations are used to introduce new extended special functions ( see $[12,13,15])$. In [13], Özarslan and Yilmaz introduced the extended Mittag-Leffler function $E_{\alpha, \beta}^{\gamma ; c}(z ; p)$ as follows:

$$
E_{\alpha, \beta}^{(\gamma ; c)}(z ; p)=\sum_{n=0}^{\infty} \frac{B(\gamma+n, c-\gamma ; p)(c)_{n}}{B(\gamma, c-\gamma) \Gamma(n \alpha+\beta)} \frac{z^{n}}{n !} .
$$

where the Mittag-Leffler functions $E_{\alpha}(z)$ and $E_{\alpha, \beta}(z)$ are defined as [see 16]:

$$
E_{\alpha}(z)=\sum_{n=0}^{\infty} \frac{z^{n}}{\Gamma(\alpha n+1)}, \quad E_{\alpha, \beta}(z)=\sum_{n=0}^{\infty} \frac{z^{n}}{\Gamma(\alpha n+\beta)} .
$$

Motivated by the works given in $[12,13,15]$, we present new extensions for the associated Laguerre polynomials in terms of the extended Beta function $B(x, y ; p)$. For this aim, we recall that 2-variable associated Laguerre polynomials (2VALP) $L_{n}^{(\alpha)}(x, y)$ are defined as [6]:

$$
L_{n}^{(\alpha)}(x, y)=\sum_{k=0}^{n} \frac{(\alpha+1)_{n}(-x)^{k} y^{n-k}}{(\alpha+1)_{k} k !(n-k) !}
$$


and specified by the following generating functions:

$$
\sum_{n=0}^{\infty} L_{n}^{(\alpha)}(x, y) t^{n}=(1-y t)^{-\alpha-1} \exp \left(\frac{-x t}{1-t y}\right) .
$$

Note that

$$
L_{n}^{(0)}(x, y)=L_{n}(x, y),
$$

where $L_{n}(x, y)$ denotes the 2-variable Laguerre polynomials (2VLP) defined as [8]:

$$
L_{n}(x, y)=n ! \sum_{k=0}^{n} \frac{(-x)^{k} y^{n-k}}{(k !)^{2}(n-k) !}
$$

and specified by the following generating functions:

$$
\sum_{n=0}^{\infty} L_{n}(x, y) \frac{t^{n}}{n !}=\exp (y t) C_{0}(x t)
$$

where $C_{0}(x)$ denotes the 0 th order Tricomi function. The $\alpha$ th order Tricomi function is defined as [16]:

$$
C_{\alpha}(x)=\sum_{k=0}^{\infty} \frac{(-x)^{k}}{k ! \Gamma(\alpha+k+1)} .
$$

Also, in particular, we note that

$$
\begin{gathered}
L_{n}^{(\alpha)}(x, 1)=L_{n}^{(\alpha)}(x), \quad L_{n}^{(\alpha)}(x, y)=y^{n} L_{n}^{(\alpha)}\left(\frac{x}{y}\right), \\
L_{n}^{(0)}(x, 1)=L_{n}(x, 1)=L_{n}(x),
\end{gathered}
$$

where $L_{n}^{(\alpha)}(x)$ and $L_{n}(x)$ denote the classical and associated Laguerre polynomials [16] respectively.

\section{An extended associated Laguerre polynomials}

In terms of the extended Beta function $B(x, y ; p)$ defined in (1), we introduce a new extension of 2-variable associated Laguerre polynomials (E2VALP), denoted by $L_{n}^{(\alpha)}(x, y ; p)$, as follows:

$$
L_{n}^{(\alpha)}(x, y ; p)=(\alpha+1)_{n} \sum_{k=0}^{n} \frac{(-x)^{k} y^{n-k} B(k+1, \alpha ; p)}{(k !)^{2}(n-k) ! B(1, \alpha)},
$$

which for $p=0$ reduces to definition (11). 
Remark 2.1. For $y=1$ in definition (19), we get the following new extension of associated Laguerre polynomials (EALP), denote by $L_{n}^{(\alpha)}(x ; p)$ and define as:

$$
L_{n}^{(\alpha)}(x ; p)=(\alpha+1)_{n} \sum_{k=0}^{n} \frac{(-x)^{k} B(k+1, \alpha ; p)}{(k !)^{2}(n-k) ! B(1, \alpha)},
$$

which for $p=0$ reduces to $L_{n}^{(\alpha)}(x)$.

In particular, we note that

$$
\begin{gathered}
L_{n}^{(\alpha)}(x, y ; p)=y^{n} L_{n}^{(\alpha)}\left(\frac{x}{y} ; p\right), \\
L_{n}^{(0)}(x, y ; 0)=L_{n}(x, y), \\
L_{n}^{(0)}(x ; 0)=L_{n}(x) .
\end{gathered}
$$

Now, we establish some representation formulae for the $\operatorname{E2VALP} L_{n}^{(\alpha)}(x, y ; p)$ in the form of the following theorems:

Theorem 2.2. The following operational representation formulas for the E2VALP $L_{n}^{(\alpha)}(x, y ; p)$ holds true:

$$
L_{n}^{(\alpha)}(x, y ; p)=(\alpha+1)_{n} \frac{y^{n}}{n !} F_{p}\left(-n, 1 ; \alpha+1 ; \frac{D_{x}^{-1}}{y}\right),
$$

where $F_{p}($.$) is the extended hypergeometric functions defined in (3) and D_{x}^{-1}$ is the inverse of the derivative operator $D_{x}=\frac{d}{d x}$ and $D_{x}^{-n}=\frac{x^{n}}{n !}$.

Proof. Using definition (19) in the L.H.S. of equation (24) and using the following relation [16]:

$$
(-n)_{k}=\frac{(-1)^{k} n !}{(n-k) !}
$$

and then in view of definition (3), we get assertion (24) of Theorem 2.2.

Corollary 2.3. The following operational representation formulae for the EALP $L_{n}^{(\alpha)}(x ; p)$ hold true:

$$
L_{n}^{(\alpha)}(x ; p)=\frac{(\alpha+1)_{n}}{n !} F_{p}\left(-n, 1 ; \alpha+1 ; D_{x}^{-1}\right),
$$

For $p=\alpha=0$ in results (24) and (26), we get the following known relations [8]:

$$
\begin{gathered}
L_{n}(x, y)=\left(y-D_{x}^{-1}\right)^{n}, \\
L_{n}(x)=\left(1-D_{x}^{-1}\right)^{n} .
\end{gathered}
$$


Theorem 2.4. The following integral representation for the $\operatorname{E2VALP} L_{n}^{(\alpha)}(x, y ; p)$ holds true:

$$
L_{n}^{(\alpha)}(x, y ; p)=\frac{\Gamma(\alpha+1)(\alpha+1)_{n}}{\Gamma(\alpha) n !} \int_{0}^{1}(1-t)^{\alpha-1} \exp \left(\frac{-p}{t(1-t)}\right) L_{n}(x t, y) d t .
$$

Proof. Using definition (19) in the L.H.S. of equation (29) and then using relation (1), we get

$$
\begin{gathered}
L_{n}^{(\alpha)}(x, y ; p)=\frac{\Gamma(\alpha+1)(\alpha+1)_{n}}{\Gamma(\alpha) n !} \int_{0}^{1}(1-t)^{\alpha-1} \exp \left(\frac{-p}{t(1-t)}\right) \\
\times \sum_{k=0}^{n} \frac{n !(-t x)^{k} y^{n-k}}{(k !)^{2}(n-k) !} d t .
\end{gathered}
$$

Using definition (14) in the R.H.S. of equation (30), we get assertion (29) of Theorem 2.4.

For $y=1$ in assertion (29) of Theorem 2.4, we get the following result.

Corollary 2.5. The following integral representation for the EALP $L_{n}^{(\alpha)}(x ; p)$ holds true:

$$
L_{n}^{(\alpha)}(x ; p)=\frac{\Gamma(\alpha+1)(\alpha+1)_{n}}{\Gamma(\alpha) n !} \int_{0}^{1}(1-t)^{\alpha-1} \exp \left(\frac{-p}{t(1-t)}\right) L_{n}(x t) d t .
$$

Next, the integral representation (2.9) will be used to derive some properties for the $\operatorname{E2VALP} L_{n}^{(\alpha)}(x, y ; p)$ as in the following theorem.

Theorem 2.6. The following differential recurrence relations for the E2VALP $L_{n}^{(\alpha)}(x, y ; p)$ hold true:

$$
\begin{gathered}
\frac{\partial}{\partial x} L_{n}^{(\alpha)}(x, y ; p)=\frac{n}{x} L_{n}^{(\alpha)}(x, y ; p)-\frac{(\alpha+n) y}{x} L_{n-1}^{(\alpha)}(x, y ; p), \\
\frac{\partial}{\partial y} L_{n}^{(\alpha)}(x, y ; p)=(\alpha+n) L_{n-1}^{(\alpha)}(x, y ; p) .
\end{gathered}
$$

Proof. Consider the following differential recurrence relations for $L_{n}(x, y)$ [8]:

$$
\begin{gathered}
\frac{\partial}{\partial x} L_{n}(x, y)=\frac{n}{x} L_{n}(x, y)-\frac{n y}{x} L_{n-1}(x, y), \\
\frac{\partial}{\partial y} L_{n}(x, y)=n L_{n-1}(x, y) .
\end{gathered}
$$


Replacing $x$ by $x t$ in relation (34) and multiplying both sides by $\frac{\Gamma(\alpha+1)(\alpha+1)_{n}}{\Gamma(\alpha) n !}(1-$ $t)^{\alpha-1} \exp \left(\frac{-p}{t(1-t)}\right)$ and then integrating the resultant equation with respect to $t$ between the limits 0 to 1 and taking in account the relation $\frac{\partial}{\partial(x t)}=\frac{\partial}{t \partial x}$, we get

$$
\begin{aligned}
& \frac{\Gamma(\alpha+1)(\alpha+1)_{n}}{\Gamma(\alpha) n !} \frac{\partial}{\partial x} \int_{0}^{1}(1-t)^{\alpha-1} \exp \left(\frac{-p}{t(1-t)}\right) L_{n}(x t, y) \\
&= \frac{\Gamma(\alpha+1)(\alpha+1)_{n} n}{\Gamma(\alpha) n ! x} \int_{0}^{1}(1-t)^{\alpha-1} \exp \left(\frac{-p}{t(1-t)}\right) L_{n}(x t, y) \\
&-\frac{y(\alpha+n) \Gamma(\alpha+1)(\alpha+1)_{n-1}}{n x \Gamma(\alpha)(n-1) !} \int_{0}^{1}(1-t)^{\alpha-1} \exp \left(\frac{-p}{t(1-t)}\right) L_{n-1}(x t, y),
\end{aligned}
$$

which on using relation (29) yields assertion (32) of Theorem 2.6.

Similarly, from relation (35) and following the same procedure leading to prove (32), we get the desired result (33) and thus the proof of Theorem 2.6 is completed.

For $y=1$ in assertion (32) of Theorem 2.6, we get the following result.

Corollary 2.7. The following differential recurrence relation for the EALP $L_{n}^{(\beta)}(x ; p)$ holds true:

$$
\frac{d}{d x} L_{n}^{(\alpha)}(x ; p)=\frac{n}{x} L_{n}^{(\alpha)}(x ; p)-\frac{(\alpha+n)}{x} L_{n-1}^{(\alpha)}(x ; p),
$$

Remark 2.8. From results (32) and (33), we get the following differential equation for the $\operatorname{E} 2 \operatorname{VALP} L_{n}^{(\alpha)}(x, y ; p)$ :

$$
\left(\frac{\partial}{\partial x}+\frac{y}{x} \frac{\partial}{\partial y}-\frac{n}{x}\right) L_{n}^{(\alpha)}(x, y ; p)=0 .
$$

\section{Generating functions}

In this section, we prove some generating functions for the E2VALP $L_{n}^{(\alpha)}(x, y ; p)$ in the form of the following theorems.

Theorem 3.1. The following generating function for the E2VALP $L_{n}^{(\alpha)}(x, y ; p)$ holds true:

$$
\sum_{n=0}^{\infty} L_{n}^{(\alpha)}(x, y ; p) \frac{(\lambda)_{n} u^{n}}{(\beta+1)_{n}}=(1-y u)^{-\lambda} \sum_{k=0}^{\infty} \frac{(\lambda)_{k} B(k+1, \alpha ; p)}{(k !)^{2} B(1, \alpha)}\left(\frac{-x u}{1-y u}\right)^{k},
$$


Proof. Using definition (19) in the L.H.S. of equation (39) and then putting $n=$ $n+k$ in the resultant equation, we get

$$
\sum_{n=0}^{\infty} L_{n}^{(\alpha)}(x, y ; p) \frac{(\lambda)_{n} u^{n}}{(\alpha+1)_{n}}=\sum_{k=0}^{\infty} \frac{(\lambda)_{k} B(k+1, \alpha ; p)(-x)^{k}}{(k !)^{2} B(1, \alpha)} \sum_{n=0}^{\infty}(\lambda+k)_{n} \frac{(y t)^{n}}{n !} .
$$

Using the following relation [16]

$$
\sum_{n=0}^{\infty}(\alpha)_{n} \frac{(t)^{n}}{n !}=(1-t)^{-\alpha}
$$

in the R.H.S. of equation (40), we get assertion (39) of Theorem 3.1.

Remark 3.2. In view of definition (3), we get the following equivalent form of generating function (39):

$$
\sum_{n=0}^{\infty} L_{n}^{(\alpha)}(x, y ; p) \frac{(\lambda)_{n} u^{n}}{(\beta+1)_{n}}=(1-y u)^{-\lambda} F_{p}\left(\lambda, 1 ; \alpha+1 ; \frac{-D_{x}^{-1} u}{1-y u}\right) .
$$

Remark 3.3. (i) For $\lambda=1$ in assertion (39) of Theorem 3.1 and in view of definition (4), we get the following generating function:

$$
\sum_{n=0}^{\infty} L_{n}^{(\alpha)}(x, y ; p) \frac{n ! u^{n}}{(\alpha+1)_{n}}=(1-y u)^{-1} \phi_{p}\left(1 ; \alpha+1 ; \frac{-x u}{1-y u}\right),
$$

where $\phi_{p}($.$) is the extended confluent hypergeometric function defined in (4).$

(ii) For $\lambda=\alpha+1$ in assertion (39) of Theorem 3.1 and in view of definition (9) we get the following generating function:

$$
\sum_{n=0}^{\infty} L_{n}^{(\alpha)}(x, y ; p) u^{n}=(1-y u)^{-\alpha-1} E_{1,1}^{(1 ; \alpha+1)}\left(\frac{-x u}{1-y u} ; p\right),
$$

where $E_{\alpha, \beta}^{(\lambda ; c)}(z ; p)$ is the extended Mittage-Leffler function defined in (9).

For $y=1$ in (39), (43) and (44), we get the following results.

Corollary 3.4. The following generating functions for the $\operatorname{EALP} L_{n}^{(\alpha)}(x ; p)$ hold true:

$$
\sum_{n=0}^{\infty} L_{n}^{(\alpha)}(x ; p) \frac{(\lambda)_{n} u^{n}}{(\beta+1)_{n}}=(1-u)^{-\lambda} \sum_{k=0}^{\infty} \frac{(\lambda)_{k} B(k+1, \alpha ; p)}{(k !)^{2} B(1, \alpha)}\left(\frac{-x u}{1-u}\right)^{k},
$$




$$
\begin{gathered}
\sum_{n=0}^{\infty} L_{n}^{(\alpha)}(x ; p) \frac{n ! u^{n}}{(\alpha+1)_{n}}=(1-u)^{-1} \phi_{p}\left(1 ; \alpha+1 ; \frac{-x u}{1-u}\right), \\
\sum_{n=0}^{\infty} L_{n}^{(\alpha)}(x ; p) u^{n}=(1-u)^{-\alpha-1} E_{1,1}^{(1 ; \alpha+1)}\left(\frac{-x u}{1-u} ; p\right) .
\end{gathered}
$$

Remark 3.5. (i) Putting $p=0$ in assertions (39) of Theorem 3.1, we get the following known generating function for the $\operatorname{VALP} L_{n}^{(\alpha)}(x, y)$ [9, p.878]:

$$
\sum_{n=0}^{\infty} L_{n}^{(\alpha)}(x, y) \frac{(\lambda)_{n} u^{n}}{(\beta+1)_{n}}=(1-y u)^{-\lambda}{ }_{1} F_{1}\left(\lambda ; \alpha+1 ; \frac{-x u}{1-y u}\right),
$$

which for $\lambda=1$ reduces to the following apparently not known generating function:

$$
\sum_{n=0}^{\infty} L_{n}^{(\alpha)}(x, y) \frac{n ! u^{n}}{(\alpha+1)_{n}}=(1-y u)^{-1} E_{1, \alpha+1}\left(\frac{-x u}{1-y u}\right),
$$

where $E_{\alpha, \beta}(z)$ is the generalized mittag-Leffler function defined in (10). (ii) For $p=0$ and $\lambda=\alpha+1$, relation (39) reduces to relation (12).

Proceeding on the same lines of proof of Theorem 3.1, we get the following result:

Theorem 3.6. The following generating function for the $\operatorname{E2VALP} L_{n}^{(\alpha)}(x, y ; p)$ hold true:

$$
\sum_{n=0}^{\infty} L_{n}^{(\alpha)}(x, y ; p) \frac{u^{n}}{(\alpha+1)_{n}}=\exp (y u) \sum_{k=0}^{\infty} \frac{(-x u)^{k} B(k+1, \alpha ; p)}{(k !)^{2} B(1, \alpha)},
$$

or equivalently

$$
\sum_{n=0}^{\infty} L_{n}^{(\alpha)}(x, y ; p) \frac{u^{n}}{(\alpha+1)_{n}}=\exp (y u) \phi_{p}\left(1 ; \alpha+1 ;-u D_{x}^{-1}\right) .
$$

For $y=1$ in assertion (50) and (51) of Theorem 3.6, we get the following results.

Corollary 3.7. The following generating function for the $\operatorname{EALP} L_{n}^{(\alpha)}(x ; p)$ hold true:

$$
\sum_{n=0}^{\infty} L_{n}^{(\alpha)}(x ; p) \frac{u^{n}}{(\alpha+1)_{n}}=\frac{\exp (u)}{B(1, \alpha)} \sum_{k=0}^{\infty} \frac{B(k+1, \alpha ; p)(-x u)^{k}}{(k !)^{2}},
$$


or equivalently

$$
\sum_{n=0}^{\infty} L_{n}^{(\alpha)}(x ; p) \frac{u^{n}}{(\alpha+1)_{n}}=\exp (u) \phi_{p}\left(1 ; \alpha+1 ;-u D_{x}^{-1}\right) .
$$

Note that, For $p=0$, relation (50) reduces to the known relation:

$$
\sum_{n=0}^{\infty} L_{n}^{(\alpha)}(x, y) \frac{u^{n}}{(\alpha+1)_{n}}=\Gamma(\alpha+a) \exp (y u) C_{\alpha}(x u) .
$$

\section{Integral formulae}

We prove the following results:

Theorem 4.1. The following integrals involving the $\operatorname{E2VALP} L_{n}^{(\beta)}(x, y ; p)$ holds true:

$$
\int_{0}^{\infty} L_{n}^{(\alpha)}(-x, y ; p) \exp (-s x) d x=\frac{(\alpha+1)_{n} y^{n}}{s} F_{p}\left(\alpha+1+n, 1 ; \alpha+1 ; \frac{1}{s}\right),
$$

where $F_{p}($.$) is the extended Hypergeometric function defined in (3).$

Proof. Using definition (19) in the L.H.S. of (55) and interchanging the order of integration and summation, we get

$$
\begin{gathered}
\int_{0}^{\infty} L_{n}^{(\alpha)}(-x, y ; p) \exp (-s x) d x=\frac{\Gamma(\alpha+1)(\alpha+1)_{n}}{\Gamma(\alpha)} \sum_{k=0}^{n} \frac{y^{n-k} B(k+1, \alpha ; p)}{(k !)^{2}(n-k) !} \\
\times \int_{0}^{\infty} \exp (-s x) x^{k} d x
\end{gathered}
$$

Using the following relation [16]:

$$
\int_{0}^{\infty} \exp (-s t) t^{\alpha-1} d t=\frac{\Gamma(\alpha)}{s^{\alpha}},
$$

in the R.H.S. of equation (56) and putting $n=n+k$ and then using relation (2), we get

$$
\begin{gathered}
\int_{0}^{\infty} L_{n}^{(\alpha)}(-x, y ; p) \exp (-s x) d x=\frac{(\alpha+1)_{n} y^{n}}{s^{\alpha+1}} \\
\times \sum_{k=0}^{n} \frac{(\alpha+1+n)_{k} B(1+k, \alpha ; p)}{k ! B(1, \alpha)}\left(\frac{1}{s}\right)^{k},
\end{gathered}
$$

which in view of definition (3), we get assertion (55) of Theorem 4.1. 
Remark 4.2. For $s=1$ in assertions (55) of Theorem 4.1, we get the following result.

$$
\int_{0}^{\infty} L_{n}^{(\alpha)}(-x, y ; p) \exp (-x) d x=(\alpha+1)_{n} y^{n} F_{p}(\alpha+1+n, 1 ; \alpha+1 ; 1),
$$

which on using the following relation [17, p.485]:

$$
F_{p}(a, b ; c ; 1)=\frac{\Gamma(c)}{\Gamma(b) \Gamma(c-b)}{ }_{2} \Psi_{1}\left[\begin{array}{cc}
(b,-1),(c-b-a,-1) ; & \\
(c-a,-2) ; & -p
\end{array}\right]
$$

in the R.H.S. gives

$$
\begin{gathered}
\int_{0}^{\infty} L_{n}^{(\alpha)}(-x, y ; p) \exp (-x) d x=\frac{\Gamma(\alpha+1+n) y^{n}}{\Gamma(\alpha)} \\
\quad \times{ }_{2} \Psi_{1}\left[\begin{array}{cc}
(1,-1),(-n-1,-1) ; & \\
(-n,-2) ; & -p
\end{array}\right],
\end{gathered}
$$

where ${ }_{2} \Psi_{1}($.$) is the Wright function (see [16]).$

For $y=1$ in results (55) and (61), we get the following results.

Corollary 4.3. The following integrals involving the $\operatorname{EALP} L_{n}^{(\alpha)}(x, y ; p)$ hold true:

$$
\begin{gathered}
\int_{0}^{\infty} L_{n}^{(\alpha)}(-x ; p) \exp (-s x) d x=\frac{(\alpha+1)_{n}}{s} F_{p}\left(\alpha+1+n, 1 ; \alpha+1 ; \frac{1}{s}\right), \\
\int_{0}^{\infty} L_{n}^{(\alpha)}(-x ; p) \exp (-x) d x=\frac{\Gamma(\alpha+1+n)}{\Gamma(\alpha)} \\
\quad \times{ }_{2} \Psi_{1}\left[\begin{array}{cc}
(1,-1),(-n-1,-1) ; & -p \\
(-n,-2) ; &
\end{array}\right] .
\end{gathered}
$$

Remark 4.4. For $p=0$ in assertion (55) of Theorem 4.1, we get the following result for the $2 \operatorname{VGLP} L_{n}^{(\alpha)}(x, y)$ :

$$
\int_{0}^{\infty} L_{n}^{(\alpha)}(-x, y) \exp (-s x) d x=\frac{(\alpha+1)_{n} y^{n}}{s}{ }_{2} F_{1}\left(\alpha+1+n, 1 ; \alpha+1 ; \frac{1}{s}\right),
$$


which on putting $s=1$ in the R.H.S. and using the following relation [16]:

$$
{ }_{2} F_{1}(a, b ; c ; 1)=\frac{\Gamma(c) \Gamma(c-b-a)}{\Gamma(c-a) \Gamma(c-b)},
$$

yields the following result:

$$
\int_{0}^{\infty} L_{n}^{(\alpha)}(-x, y) \exp (-x) d x=\frac{(\alpha+1)_{n} \Gamma(\alpha+1) \Gamma(-n-1) y^{n}}{\Gamma(-n) \Gamma(\alpha)} .
$$

Note that, for $y=1$ in relations (64) and (66), we get the following results.

$$
\begin{gathered}
\int_{0}^{\infty} L_{n}^{(\alpha)}(-x) \exp (-s x) d x=\frac{(\alpha+1)_{n}}{s}{ }_{2} F_{1}\left(\alpha+1+n, 1 ; \alpha+1 ; \frac{1}{s}\right) \\
\int_{0}^{\infty} L_{n}^{(\alpha)}(-x) \exp (-x) d x=\frac{(\alpha+1)_{n} \Gamma(\alpha+1) \Gamma(-n-1)}{\Gamma(-n) \Gamma(\alpha)}
\end{gathered}
$$

Theorem 4.5. The following integral involving the E2VALP $L_{n}^{(\beta)}(x, y ; p)$ holds true:

$$
\begin{gathered}
\frac{1}{(\alpha+1)_{n}} \int_{0}^{\infty} \int_{0}^{\infty} L_{n}^{(\alpha)}(x, y ; p) \exp (-r x-s y) d x d y \\
=\frac{1}{r s^{n+1}} \Phi_{p}\left(1 ; \alpha+1 ;-\frac{s}{r}\right) .
\end{gathered}
$$

Proof. Using definition (19) in the L.H.S. of (69) and interchanging the order of integration and summation, we get

$$
\begin{aligned}
& \frac{1}{(\alpha+1)_{n}} \int_{0}^{\infty} \int_{0}^{\infty} L_{n}^{(\alpha)}(x, y ; p) \exp (-r x-s y) d x d y=\frac{\Gamma(\alpha+1)}{\Gamma(\alpha)} \\
\times & \sum_{k=0}^{n} \frac{(-1)^{k} B(k+1, \alpha ; p)}{(k !)^{2}(n-k) !} \int_{0}^{\infty} \exp (-r x) x^{k} d x \int_{0}^{\infty} \exp (-s y) y^{n-k} d y,
\end{aligned}
$$

which on using relation (57) in the R.H.S. and then in view of definition (4) yields assertion (69) of Theorem 4.5.

Remark 4.6. For $p=0$ in assertion (69) of Theorem 4.5, we get the following result for the 2 VALP $L_{n}^{(\alpha)}(x, y)$ :

$$
\begin{gathered}
\frac{1}{(\alpha+1)_{n}} \int_{0}^{\infty} \int_{0}^{\infty} L_{n}^{(\alpha)}(x, y) \exp (-r x-s y) d x d y \\
=\frac{1}{r s^{n+1}{ }_{1} F_{1}\left(1 ; \alpha+1 ;-\frac{s}{r}\right)}
\end{gathered}
$$

which for $\alpha=0$ yields the following result for the $2 \operatorname{VLP} L_{n}(x, y)$ :

$$
\frac{1}{n !} \int_{0}^{\infty} \int_{0}^{\infty} L_{n}(x, y) \exp (-r x-s y) d x d y=\frac{1}{r s^{n+1}} \exp \left(-\frac{s}{r}\right) \text {. }
$$




\section{Summation formulae}

We prove the following summation formulae by using integral representation (27):

Theorem 5.1. The following summation formulae for the $\operatorname{E2VALP} L_{n}^{(\alpha)}(x, y ; p)$ hold true:

$$
\begin{aligned}
L_{n}^{(\alpha)}\left(\frac{x}{1-u y^{-1}}, y ; p\right) & =\left(1-u y^{-1}\right)^{-n} \sum_{k=0}^{n} L_{n-k}^{(\alpha)}(x, y ; p) \frac{(-\alpha-n)_{k} u^{k}}{k !}, \\
L_{n}^{(\alpha)}(x y, z ; p) & =\sum_{k=0}^{n} \frac{(\alpha+1)_{n}[z(1-y)]^{n-k} y^{k}}{(\alpha+1)_{k}(n-k) !} L_{k}^{(\alpha)}(x, z ; p) .
\end{aligned}
$$

Proof. To prove (73), consider the following relation [11, p.5]:

$$
\left(1-u y^{-1}\right)^{n} L_{n}\left(\frac{x}{1-u y^{-1}}, y\right)=\sum_{k=0}^{\infty} L_{n-k}(x, y) \frac{(-n)_{k} u^{k}}{k !} .
$$

Replacing $x$ by $x t$ in (75) and multiplying both sides by $(1-t)^{\alpha-1} \exp \left(\frac{-p}{t(1-t)}\right)$ and integrating the resultant equation with respect to $t$ between the limits 0 to 1 , we get

$$
\begin{gathered}
\left(1-u y^{-1}\right)^{n} \int_{0}^{1}(1-t)^{\alpha-1} \exp \left(\frac{-p}{t(1-t)}\right) L_{n}\left(\frac{x t}{1-u y^{-1}}, y\right) d t \\
=\sum_{k=0}^{\infty} \frac{(-n)_{k} u^{k}}{k !} \int_{0}^{1}(1-t)^{\alpha-1} \exp \left(\frac{-p}{t(1-t)}\right) L_{n-k}(x t, y) d t
\end{gathered}
$$

which in view of relation (29) and after some simplifications yields assertion (73) of Theorem 5.1.

Similarly, proceeding on the same lines of proof of Theorem 5.1 and using the following relation [7, p.879] (for $\alpha=0$ ):

$$
L_{n}(x y, z)=\sum_{k=0}^{n} \frac{n ![z(1-y)]^{n-k} y^{k}}{k !(n-k) !} L_{k}(x, z),
$$

we get assertions (74) of Theorem 5.1, thus the proof of Theorem 5.1 is completed.

For $y=1$ and $z=1$ in assertions (73) and (74) of Theorem 5.1 respectively, we get the following results: 
Corollary 5.2. The following summation formulae for the $\operatorname{EALP} L_{n}^{(\alpha)}(x ; p)$ hold true:

$$
\begin{aligned}
L_{n}^{(\alpha)}\left(\frac{x}{1-u} ; p\right) & =(1-u)^{-n} \sum_{k=0}^{n} L_{n-k}^{(\alpha)}(x ; p) \frac{(-\alpha-n)_{k} u^{k}}{k !}, \\
L_{n}^{(\alpha)}(x y ; p) & =\sum_{k=0}^{n} \frac{(\alpha+1)_{n}(1-y)^{n-k} y^{k}}{(\alpha+1)_{k}(n-k) !} L_{k}^{(\alpha)}(x ; p) .
\end{aligned}
$$

Remark 5.3. For $p=0$ in assertions (73) and (74) of Theorem 5.1, we get the following known results given in [10] and [9] respectively:

$$
\begin{aligned}
L_{n}^{(\alpha)}\left(\frac{x}{1-u y^{-1}}, y\right) & =\left(1-u y^{-1}\right)^{-n} \sum_{k=0}^{n} L_{n-k}^{(\alpha)}(x, y) \frac{(-\alpha-n)_{k} u^{k}}{k !}, \\
L_{n}^{(\alpha)}(x y, z) & =\sum_{k=0}^{n} \frac{(\alpha+1)_{n}[z(1-y)]^{n-k} y^{k}}{(\alpha+1)_{k}(n-k) !} L_{k}^{(\alpha)}(x, z) .
\end{aligned}
$$

\section{Concluding remarks}

In view of definition (16), we extend the $\alpha$ th order Tricomi function as follows:

$$
C_{\alpha}(x ; p)=\frac{1}{\Gamma(\alpha+1)} \sum_{k=0}^{\infty} \frac{(-x)^{k} B(k+1, \alpha ; p)}{(k !)^{2} B(1, \alpha)},
$$

which for $p=0$ reduces to definition (16).

We recall that the 2-variable Hermite-Tricomi functions (2VHTF) ${ }_{H} C_{\alpha}(x, y)$ are defined as (see [7]):

$$
{ }_{H} C_{\alpha}(x, y)=\sum_{k=0}^{\infty} \frac{(-1)^{k} H_{k}(x, y)}{k ! \Gamma(\alpha+k+1)},
$$

where $H_{n}(x, y)$ denotes the 2-variable Hermite-Kampé de Fériet polynomials (2VHKdFP) defined by [2]:

$$
H_{n}(x, y)=n ! \sum_{k=0}^{\left[\frac{n}{2}\right]} \frac{y^{k} x^{n-2 k}}{k !(n-2 k) !}
$$

and satisfied the following relation:

$$
t^{n} H_{n}(x, y)=H_{n}\left(x t, y t^{2}\right) .
$$


Now, by using definition (82) and in view of definition (83), we introduce a new extended 2-variable Hermite-Tricomi functions (E2VHTF) ${ }_{H} C_{\alpha}(x, y ; p)$ as follows:

$$
{ }_{H} C_{\alpha}(x, y ; p)=\frac{1}{\Gamma(\alpha+1)} \sum_{k=0}^{\infty} \frac{(-1)^{k} H_{k}(x, y) B(k+1, \alpha ; p)}{(k !)^{2} B(1, \alpha)},
$$

which for $p=0$ reduces to definition (83).

Next, definition (86) will be used to prove the following generating functions for the $\operatorname{E2VALP} L_{n}^{(\beta)}(x, y ; p)$ :

Theorem 6.1. The following generating function for the $\operatorname{E2VALP} L_{n}^{(\alpha)}(x, y ; p)$ holds true:

$$
\sum_{n=0}^{\infty} L_{2 n}^{(\alpha)}(x, y ; p) \frac{(2 n) ! u^{n}}{n !(\alpha+1)_{2 n}}=\Gamma(\alpha+1) \exp \left(y^{2} u\right){ }_{H} C_{\alpha}\left(2 x y u, x^{2} u ; p\right) .
$$

Proof. Consider the following relation [3]:

$$
\sum_{n=0}^{\infty} L_{2 n}(x, y) \frac{u^{n}}{n !}=\exp \left(y^{2} u\right){ }_{H} C_{0}\left(2 x y u, x^{2} u\right) .
$$

Using definition (83) to expand the R.H.S. of equation (88) and then making use of relation (85), we obtain

$$
\sum_{n=0}^{\infty} L_{2 n}(x, y) \frac{u^{n}}{n !}=\exp \left(y^{2} u\right) \sum_{k=0}^{\infty} \frac{(-x)^{k} H_{k}(2 y u, u)}{(k !)^{2}},
$$

which on replacing $x$ by $x t$ and multiplying both sides by $(1-t)^{\alpha-1} \exp \left(\frac{-p}{t(1-t)}\right)$ and integrating the resultant equation with respect to $t$ between the limits 0 to 1 and then using relations (19) and (1) in the L.H.S. and R.H.S. respectively gives

$$
\begin{gathered}
\sum_{n=0}^{\infty} L_{2 n}^{(\alpha)}(x, y ; p) \frac{(2 n) ! u^{n}}{n !(\alpha+1)_{2 n}} \\
=\exp \left(y^{2} u\right) \sum_{k=0}^{\infty} \frac{(-1)^{k} H_{k}\left(2 x y u, x^{2} u\right) B(k+1, \alpha ; p)}{(k !)^{2} B(1, \alpha)} .
\end{gathered}
$$

which in view of definition (86) yields assertion (87) of Theorem 6.1.

For $y=1$ in assertion (87) of Theorem 6.1, we get the following result: 
Corollary 6.2. The following generating function for the $\operatorname{EALP} L_{n}^{(\alpha)}(x ; p)$ holds true:

$$
\sum_{n=0}^{\infty} L_{2 n}^{(\alpha)}(x ; p) \frac{(2 n) ! u^{n}}{n !(\alpha+1)_{2 n}}=\Gamma(\alpha+1) \exp (u){ }_{H} C_{\alpha}\left(2 x u, x^{2} u ; p\right) .
$$

Remark 6.3. For $p=0$ in assertion (87) of Theorem 6.1, we get the following generating function for the $\operatorname{VALP} L_{n}^{(\alpha)}(x, y)$ :

$$
\sum_{n=0}^{\infty} L_{2 n}^{(\alpha)}(x, y) \frac{(2 n) ! u^{n}}{n !(\alpha+1)_{2 n}}=\Gamma(\alpha+1) \exp \left(y^{2} u\right)_{H} C_{\alpha}\left(2 x y u, x^{2} u\right)
$$

which for $\alpha=0$ reduces to relation (88).

Remark 6.4. Using the following generating function [7]:

$$
\begin{gathered}
\sum_{n=0}^{\infty} L_{n}(x, y) H_{n}(z, w) \frac{u^{n}}{n !} \\
=\exp \left(y z t+w(y u)^{2}\right){ }_{H} C_{0}\left(x z u+2 x y w u^{2}, w(x u)^{2}\right),
\end{gathered}
$$

and proceeding on the same lines of proof of Theorem 3.1 and using definition (86), we get the following result:

Theorem 6.5. The following bilateral generating function for the E2VALP $L_{n}^{(\alpha)}(x, y ; p)$ holds true:

$$
\begin{gathered}
\sum_{n=0}^{\infty} L_{n}^{(\alpha)}(x, y ; p) H_{n}(z, w) \frac{u^{n}}{(\alpha+1)_{n}} \\
=\Gamma(\alpha+1) \exp \left(y z t+w(y u)^{2}\right){ }_{H} C_{\alpha}\left(x z u+2 x y w u^{2}, w(x u)^{2} ; p\right) .
\end{gathered}
$$

For $y=1$ in assertion (94) of Theorem 6.5, we get the following result:

Corollary 6.6. The following generating function for the $\operatorname{EALP} L_{n}^{(\alpha)}(x ; p)$ holds true:

$$
\begin{gathered}
\sum_{n=0}^{\infty} L_{n}^{(\alpha)}(x ; p) H_{n}(z, w) \frac{u^{n}}{(\alpha+1)_{n}} \\
=\Gamma(\alpha+1) \exp \left(z t+w(u)^{2}\right){ }_{H} C_{\alpha}\left(x z u+2 x w u^{2}, w(x u)^{2} ; p\right) .
\end{gathered}
$$


Remark 6.7. For $p=0$ in assertion (94) of Theorem 6.5, we get the following generating function for the $\operatorname{VALP} L_{n}^{(\alpha)}(x, y)$ :

$$
\begin{gathered}
\sum_{n=0}^{\infty} L_{n}^{(\alpha)}(x, y) H_{n}(z, w) \frac{u^{n}}{(\alpha+1)_{n}} \\
=\Gamma(\alpha+1) \exp \left(y z t+w(y u)^{2}\right)_{H} C_{\alpha}\left(x z u+2 x y w u^{2}, w(x u)^{2}\right),
\end{gathered}
$$

which for $\alpha=0$ reduces to relation (93).

\section{Bibliography}

[1] P. Agarwal, J. Choi and S. Jain, Extended hypergeometric functions of two and three variables, Commun. Korean Math. Soc. 30(4) (2015) 403-414.

[2] P. Appell and J. Kamp, é de Fériet, Fonctions hypergéométriques et hypersphériques: Polynômes d' Hermite, Gauthier-Villars, Paris, 1926.

[3] D. Babusci, G. Dattoli, K. Gorska and K. A. Penson, Lacunary generating functions for Laguerre polynomials, Sém. Lothar. Combin. 76 (2017) Article B76b, 1-19.

[4] M. A. Chaudhry, A. Qadir, M. Rafique and S. M. Zubair, Extension of Euler's beta function, J. Comput. Appl. Math. 78 (1997) 19-32.

[5] M. A. Chaudhry, A. Qadir, H. M. Srivastava and R. B. Paris, Extended hypergeometric and confluent hypergeometric functions, Appl. Math. Comput. 159 (2004) 589-602.

[6] G. Dattoli, Generalized polynomials, operational identities and their applications, $J$. Comput. Appl. Math. 118(1) (2000) 111-123.

[7] G. Dattoli, Bilateral generating functions and operational methods, J. Math. Anal. Appl. 269 (2002) 716-725.

[8] G. Dattoli and A. Torre, Exponential operators, quasi-monomials and generalized polynomials, Radiat. Phys. Chem. 57(1) (2000) 21-26.

[9] K. S. Dhounsi and Yasmeen, General study on Laguerre polynomials of two variable, Int. J. Comput. Appl. Math. 12(3) (2017) 877-885.

[10] S. Khan and G. Yasmin, On generating function for the two variables modified Laguerre polynomials, Bull. Math. Soc. Sci. Math Roumanie Tome 45(93) (2002) 29-37.

[11] S. Khan and G. Yasmin, Lie-Theoretic generating relations of two variables Laguerre polynomials, Rep. Math. Phys. 51 (2003) 1-7.

[12] M. A. Özarslan and E. Özergin, Some generating relations for extended hypergeometric functions via generalized fractional derivative operator, Math. Comput. Modelling 52 (2010) 1825-1833. 
[13] M. A. Özarslan and B. Yilmaz, The extended Mittag-Leffler function and its properties, J. Inequ. Appl. 85(1) (2014) Article number: 85.

[14] E. Özergin, M. A. Özarslan and A. Altin, Extension of gamma, beta and hypergeometric functions, J. Comput. Appl. Math. 235 (2011) 4601-4610.

[15] R. K. Parmar, J. Choi and S. D. Purohit, Further generalization of the extended Hurwitz-Lerch Zeta functions, Bol. Soc. Paran. Mat. 37(1) (2019) 177-190.

[16] E. D. Rainville, Special Functions, Macmillan, New York, 1960.

[17] D. K. Singh, On extended hypergeometric function, Thai J. Math. 15(2) (2017) 483490.

Received May 9, 2020; revised October 12, 2020; accepted November 25, 2020.

\section{Author information}

Ahmed Ali Al-Gonah, Department of Mathematics, Faculty of Science, Aden University, Aden, Yemen.

E-mail: gonah1977@yahoo.com 